\title{
p53 protein expression affected by TP53 polymorphism is associated with the biological behavior and prognosis of low rectal cancer
}

\author{
GUANGZHE ZHANG $^{1,2}$, QIAN XU ${ }^{1,2}$, ZEYANG WANG $^{1,2}$, LIPING SUN $^{1,2}$, ZHI LV $^{1,2}$, \\ JINGWEI LIU ${ }^{1,2}$, CHENGZHONG XING ${ }^{1,2}$ and YUAN YUAN ${ }^{1,2}$ \\ ${ }^{1}$ Department of Tumor Etiology and Screening, Cancer Institute and General Surgery, The First Hospital of \\ China Medical University; ${ }^{2}$ Key Laboratory of Cancer Etiology and Prevention in Liaoning Education Department, \\ The First Hospital of China Medical University, Shenyang, Liaoning 110001, P.R. China
}

Received March 21, 2018; Accepted November 29, 2018

DOI: $10.3892 / 01.2019 .10999$

\begin{abstract}
Low rectal cancer is a subtype of colorectal cancer at a special anatomic site with distinct biological behavior. TP53 is one of the most important cancer suppressor genes, and its structural variation and abnormal expression has been revealed to be associated with multiple cancer types. However, to the best of our knowledge, the association of $\mathrm{p} 53$ protein expression with its gene polymorphism, biological behavior and prognosis in low rectal cancer has not been clarified. Therefore, the current study aimed to explore these associations. In the present study, 347 patients with low rectal cancer and 353 controls were enrolled. Kompetitive Allele-Specific Polymerase Chain Reaction was used to detect five polymorphic sites of the TP53 gene (rs1042522, rs12947788, rs1625895, rs2909430 and rs12951053), while immunohistochemistry was used to detect the protein expression of TP53. The associations between p53 protein expression and TP53 polymorphism, biological behavior and prognosis in low rectal cancer were systematically analyzed. In low rectal cancer, p53 protein expression was markedly higher in TP53 rs1042522 mutant carriers compared with that in other genotypes where expression was higher in poorly differentiated, III-IV phase and T3-4 phase tumors, and in III-IV phase female patients. The survival time of patients with low p53 protein expression was evidently longer in females, non-smokers and patients $>60$ years old. In summary, $\mathrm{p} 53$ protein expression was identified to be affected by TP53 rs1042522 polymorphism, and was associated with the biological behavior and prognosis of low rectal cancer.
\end{abstract}

Correspondence to: Dr Yuan Yuan, Department of Tumor Etiology and Screening, Cancer Institute and General Surgery, The First Hospital of China Medical University, 155 NanjingBei Street, Shenyang, Liaoning 110001, P.R. China

E-mail: yuanyuan@cmu.edu.cn

Key words: cellular tumor antigen p53, expression, polymorphism, low rectal cancer, prognosis
TP53 rs1042522 and the associated protein expression could be used as indicators for biological behavior and prognosis in low rectal cancer.

\section{Introduction}

Low rectal cancer (LRC) is located in an area that is $6-8 \mathrm{~cm}$ away from the rectum (1). LRC is a type of colorectal cancer that occurs at a specific anatomical site and exhibits a specific biological behavior. Compared with middle and upper rectal cancer, LRC possesses different pathological types, clinical outcomes and surgical options $(2,3)$. Despite advancements in treatment options for LRC and an improved understanding of its biological characteristics, LRC remains a challenge to human health due to its high local recurrence risk (4). The accurate classification of molecular phenotype may significantly contribute to monitoring the biological behavior of LRC and improve the personalized prognosis for the disease.

The TP53 gene, located at the 17p13.1 locus of the short arm of the human chromosome, covers an overall length of $16-20 \mathrm{~kb}$ and consists of 11 exons and 10 introns (5). The TP53 gene encodes an intranuclear phosphorylated protein that consists of 393 amino acids, with a 25-kb mRNA transcription product $(6,7)$. Wild-type TP53 is a cancer suppressor gene that serves a crucial role in multiple cellular processes, including the cell cycle, cell apoptosis, cell aging, gene stability and the inhibition of angiogenesis (8-10). By contrast, mutated TP53 can stimulate cell division and function as an oncogene. It is well understood that mutation of the TP53 gene and dysfunction of the TP53 pathway is a characteristic hallmark of various types of human malignancy (11). In addition to mutations, polymorphisms in the TP53 gene may occur in coding and non-coding sequences. According to previous studies, at least eight polymorphic sites have been detected in the promoter region of the TP53 gene, as well as in the first, second, third, sixth, seventh and tenth intron regions, and in the seventh exon region. Among these polymorphisms, three polymorphic sites have been associated with genetic susceptibility to multiple cancer types. These include a CD72 Arg/Pro polymorphism, a repetitive sequence inserted in $16 \mathrm{bp}$ of the third intron region 
and a polymorphism of the restriction enzyme digestion site of MspI in the sixth intron (12-14). As one of these functional TP53 single nucleotide polymorphisms (SNPs), the CD72 Arg/Pro polymorphism (rs1042522) has been studied in colon cancer. One study reported that there was no evident association between rs1042522 and colorectal cancer (15), while two study groups identified that the rs1042522 polymorphic genotype was associated with increased colon cancer risk $(16,17)$.

With structural variation of the TP53 gene, abnormal protein expression of p53 has also been revealed to be associated with multiple cancer types, including colorectal cancer. A literature review revealed that the overexpression of p53 is an independent predictor for cancer survival (18). However, another study did not identify a prognostic value of p53 in colorectal cancer (19). A further study demonstrated that p53 protein expression is associated with short-term prognosis in colorectal cancer, since a significant association between p53 expression and rectal carcinoma was identified and the percentage of p53 positive cells was associated with clinicopathological variables (20).

Although the association between p53 and colorectal cancer has been studied for a number of years, the majority of previous studies failed to investigate colorectal cancer based on the position of the lesion site or only divided colorectal cancer into colon cancer and rectum cancer. Furthermore, the conclusions of these previous studies have been contradictory. To the best of our knowledge, the association between p53 and LRC has not been investigated in previous studies. Therefore, it remains unclear whether p53 protein expression is associated with TP53 gene polymorphisms in LRC, and whether p53 protein expression is associated with the biological behavior and prognosis of LRC.

Based on patients with or without LRC, associations between the five most common polymorphic sites of the TP53 gene (rs1042522, rs12947788, rs1625895, rs2909430 and rs12951053) and p53 protein expression were investigated in the present study. In addition, the associations between p53 protein expression and biological behavior and the prognosis of LRC were systematically studied. The overall aim of the current study was to provide information that may be useful for the development of individualized therapeutic strategies prior to surgery, and to improve the biological behavior and prognosis of patients with LRC in clinical practice.

\section{Materials and methods}

Patients. The current study was approved by the Medical Ethics Committee of the First Hospital of China Medical University (Shenyang, China) and written informed consent for use of samples was obtained from all participants. A total of 347 patients diagnosed with LRC (within $8 \mathrm{~cm}$ from the anal verge), treated by surgery at the Department of Anus and Intestine Surgery of the First Hospital of China Medical University (Shenyang, China) between December 2011 and June 2016, were included in the present study. A total of 353 patients with an anal benign lesion, but no colorectal cancer, as determined by colonoscopy and rectal examination, were hospitalized during the same period and used as controls. The mean ages of patients with LRC and patients with an anal benign lesion were $61.4 \pm 11.0$ and
Table I. Sequences of the primers used for the Kompetitive allele specific polymerase chain reaction.

SNPs Primer sequences

rs 1042522

Forward

Reverse

GGGTCTTACGGTCTCCGACGAGGGG

rs 12947788

Forward

GCACCGGGGACGTGGTCGTCGAGGA

Reverse

rs 1625895

Forward

Reverse

rs 2909430

Forward

CCTCTGCTTGCCTCTGACCCCTGGG

Reverse

rs 12951053

Forward

CCACCTCTTACCGATTTCTTCCATA

Reverse

ATTCCCACCAACAGTCACCGGGAGG

CCACTCGTCATCCCCCCGAAAGAGG

GATCACCCAACGTCCTCCACGAATG

GTACAAACAAAGAAACGACGGCAGA

CTGGGCCCACCTCTTACCGATTTCT CCATACTACTACCCATCCACCTCTC

Table II. Thermocycling conditions for the polymerase chain reaction.

\begin{tabular}{lccc}
\hline Steps & Temperature & Duration & $\begin{array}{c}\text { Number } \\
\text { of cycles }\end{array}$ \\
\hline 1 & & & \\
Activation & $94^{\circ} \mathrm{C}$ & $15 \mathrm{~min}$ & 1 \\
2 & & & \\
Denaturation & $94^{\circ} \mathrm{C}$ & $20 \mathrm{sec}$ & 10 \\
Annealing/Elongation & $55-61^{\circ} \mathrm{C}$ & $60 \mathrm{sec}$ & \\
3 & & & \\
Denaturation & $94^{\circ} \mathrm{C}$ & $20 \mathrm{sec}$ & 26 \\
Annealing/Elongation & $55^{\circ} \mathrm{C}$ & $60 \mathrm{sec}$ & \\
\hline
\end{tabular}

$59.6 \pm 14.4$ years, respectively. The sex distribution (male vs female) in patients group and control group were 203:144 and 185:168, respectively.

The inclusion criteria were as follows: i) Rectal cancer diagnosed within $8 \mathrm{~cm}$ of the anal verge; and ii) age $>18$ years old. The clinical diagnostic criteria for LRC were defined according to the literature (21). The exclusion criteria were as follows: i) Patients with an immune system disease; ii) patients with an infectious disease; iii) patients with primary tumors on other visceral organs prior to surgery; and iv) patients who received neoadjuvant chemoradiotherapy prior to surgery.

Sample and patient history collection. The peripheral blood of each individual included in the present study was collected prior to surgery for patients or prior to colonoscopy for controls for genomic DNA extraction. Each sample was immediately frozen and kept at $-80^{\circ} \mathrm{C}$ until further use. The basic information of each 
Table III. Associations between polymorphisms of the TP53 gene and p53 protein expression.

\begin{tabular}{|c|c|c|c|c|c|c|c|}
\hline \multirow{2}{*}{$\begin{array}{l}\text { p53 protein } \\
\text { expression }\end{array}$} & \multicolumn{3}{|c|}{ Genotype } & \multirow{2}{*}{$\begin{array}{c}\begin{array}{c}\text { Heterozygous } \\
\text { vs.wild-type }\end{array} \\
\text { P-value }\end{array}$} & \multirow{2}{*}{$\begin{array}{l}\begin{array}{l}\text { Mutant vs. } \\
\text { wild-type }\end{array} \\
\text { P-value }\end{array}$} & \multirow{2}{*}{$\frac{\begin{array}{c}\text { Dominant } \\
\text { model }\end{array}}{\text { P-value }}$} & \multirow{2}{*}{$\frac{\begin{array}{c}\text { Recessive } \\
\text { model }\end{array}}{\text { P-value }}$} \\
\hline & Wild-type, $\mathrm{n}$ & Heterozygous, $\mathrm{n}$ & Mutant, $\mathrm{n}$ & & & & \\
\hline rs1042522 & & & & 0.027 & 0.239 & 0.032 & 0.905 \\
\hline Positive & 64 & 135 & 49 & & & & \\
\hline Negative & 37 & 43 & 19 & & & & \\
\hline rs12947788 & & & & 0.203 & 0.990 & 0.278 & 0.659 \\
\hline Positive & 97 & 125 & 26 & & & & \\
\hline Negative & 45 & 42 & 12 & & & & \\
\hline rs1625895 & & & & 0.280 & & & \\
\hline Positive & 221 & 27 & & & & & \\
\hline Negative & 92 & 7 & & & & & \\
\hline rs2909430 & & & & 0.204 & & & \\
\hline Positive & 216 & 32 & & & & & \\
\hline Negative & 91 & 8 & & & & & \\
\hline rs12951053 & & & & 0.154 & 0.787 & 0.191 & 0.905 \\
\hline Positive & 116 & 108 & 24 & & & & \\
\hline Negative & 54 & 35 & 10 & & & & \\
\hline
\end{tabular}

A

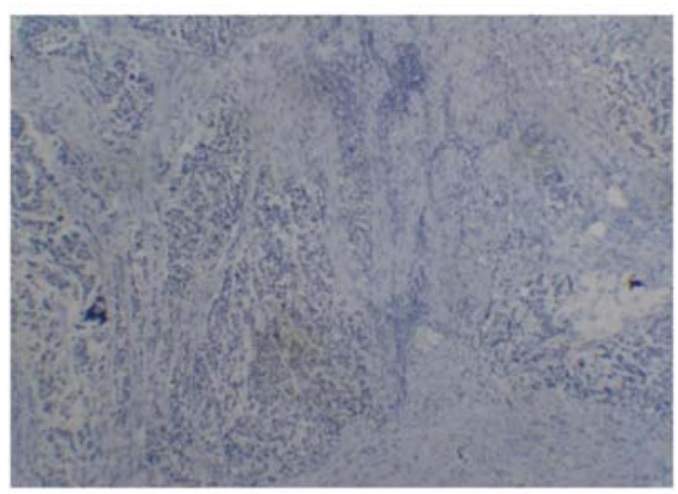

C

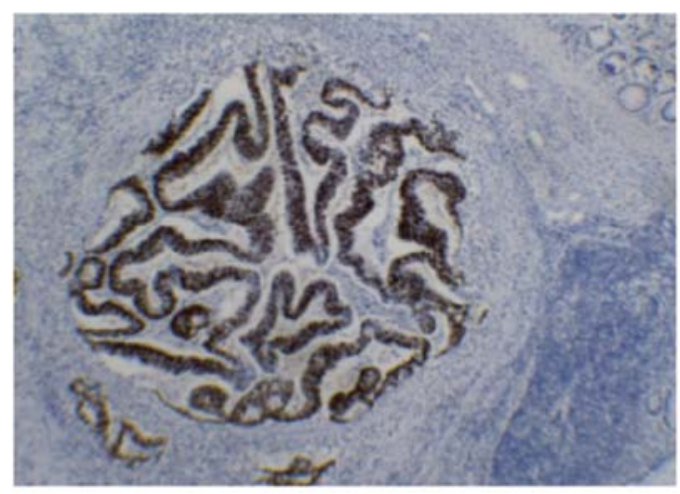

B

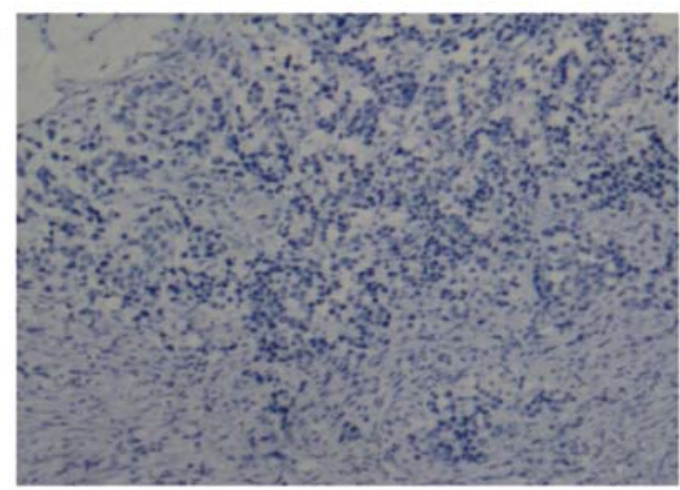

D

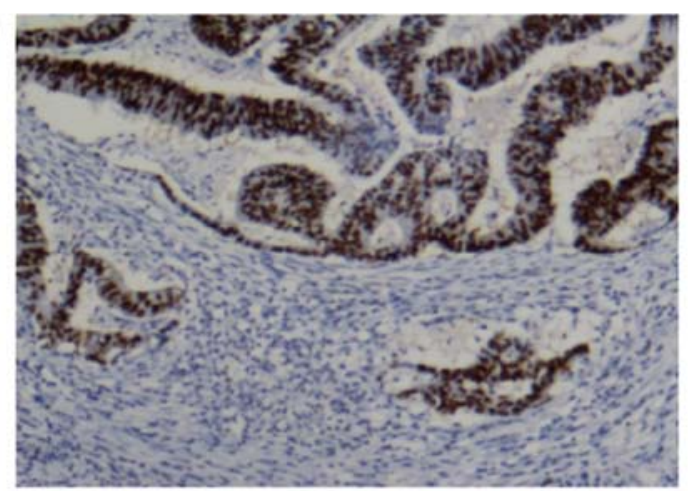

Figure 1. Determination of p53 protein expression by immunohistochemistry. (A) p53 negative expression (magnification, $\mathrm{x} 40$ ). (B) p53 negative expression (magnification, $x 200$ ). (C) p53 positive expression (magnification, $x 40$ ). (D) p53 positive expression (magnification, $x 200$ ).

individual was collected using a questionnaire, which included their sex, age, and smoking status and alcohol consumption. Data regarding the Tumor-Node-Metastasis (TNM) system classification, depth of invasion, growth pattern, histological type, paracancerous lymphocyte infiltration status, peripheral ganglion violation status, cancer embolus in vascularization, lymph node metastasis and implantation in extra nodes were extracted from the medical records of patients with LRC. The overall survival (OS) of individuals following diagnosis or treatment was assessed until August 2016. 
Table IV. Clinical characteristics and overall survival time of patients with low rectal cancer.

\begin{tabular}{|c|c|c|c|c|}
\hline Characteristic & Low rectal cancer, $\mathrm{n}$ & Mortality, n & Median survival time & P-value \\
\hline Sex & 304 & 44 & & 0.193 \\
\hline Male & 178 & 29 & 38.504 & \\
\hline Female & 126 & 15 & 38.908 & \\
\hline Age, years & 304 & 44 & & 0.827 \\
\hline$\leq 60$ & 138 & 20 & 39.656 & \\
\hline$>60$ & 166 & 24 & 38.362 & \\
\hline TNM stage & 303 & 44 & & $3.246 \times 10^{-10}$ \\
\hline I-II & 181 & 8 & 43.197 & \\
\hline III-IV & 122 & 36 & 33.081 & \\
\hline Depth of infiltration & 304 & 44 & & 0.003 \\
\hline $\mathrm{T} 1+\mathrm{T} 2$ & 87 & 4 & 42.988 & \\
\hline $\mathrm{T} 3+\mathrm{T} 4$ & 217 & 40 & 37.261 & \\
\hline Lymph node metastasis & 303 & 44 & & $2.406 \times 10^{-11}$ \\
\hline Negative & 193 & 8 & 43.288 & \\
\hline Positive & 110 & 36 & 32.490 & \\
\hline Histological type & 304 & 44 & & $4.1911 \times 10^{-8}$ \\
\hline Well-differentiated & 195 & 13 & 42.412 & \\
\hline Poorly differentiated & 109 & 31 & 32.060 & \\
\hline Peripheral lymphocyte infiltration & 283 & 41 & & 0.619 \\
\hline Negative & 24 & 4 & 40.125 & \\
\hline Positive & 259 & 37 & 35.165 & \\
\hline Peripheral ganglion violation & 266 & 39 & & 0.002 \\
\hline Negative & 72 & 4 & 38.586 & \\
\hline Positive & 194 & 35 & 32.952 & \\
\hline Vascular cancer embolus & 293 & 44 & & 0.001 \\
\hline Negative & 222 & 25 & 40.574 & \\
\hline Positive & 71 & 19 & 33.467 & \\
\hline Implantation in extra nodes & 264 & 39 & & $<0.001$ \\
\hline Negative & 246 & 30 & 35.886 & \\
\hline Positive & 18 & 9 & 23.202 & \\
\hline
\end{tabular}

TNM, Tumor-Node-Metastasis.

Candidate TP53 gene SNP selection. To explore the association between TP53 gene polymorphisms and p53 protein expression, a total of 5 SNPs (rs1042522, rs12947788, rs1625895, rs2909430 and rs12951053) with a minimum allele frequency $<5 \%$ in the Chinese population were selected based on the tagging information from the NCBI dbSNP (https://www.ncbi. nlm.nih.gov/snp) and International HapMap Project (www. hapmap.org) in 2016.

Kompetitive allele-specific polymerase chain reaction (KASP ${ }^{T M}$ genotyping assay. Genomic DNA was prepared from peripheral blood mononuclear cells collected from patients using the QIAamp DNA Blood Mini kit (Qiagen China Co., Ltd., Shanghai, China) according to the manufacture's protocol and stored at $-80^{\circ} \mathrm{C}$. SNP genotyping was performed applying KASP with an SNPLine platform (LGC Genomics). The steps of the PCR were as follows: i) The extracted DNA samples were diluted in $30 \mu 1$ TE buffer (concentration $\geq 60 \mathrm{ng} / \mu \mathrm{l}$ ) in 96 -well plates, and transferred into 384-well plates and 1536-well plates by Replikator (final concentration $\sim 10 \mathrm{ng} / \mu \mathrm{l})$; ii) the 1536 -well plates containing DNA samples were dried in an oven at $65^{\circ} \mathrm{C}$ for $30 \mathrm{~min}$; iii) the PCR reaction system $(1 \mu \mathrm{l})$ was constructed and the sequences of primers used were presented in Table I; iv) plates with reaction system were sealed and centrifuged at 12,000 x g; v) PCR was performed in water bath after centrifugation according to the thermal cycling conditions presented in Table II; vi) plates with completed reaction were cooled down and read with a microplate reader Pherastar (BMG Labtech $\mathrm{GmbH}$ ); and vii) additional PCR would be performed to double check the genotyping results if necessary.

Immunohistochemistry assay. Tissue specimens were fixed with $10 \%$ formalin at room temperature for $24 \mathrm{~h}$ and embedded 
Table V. Overall association analysis between p53 protein expression and characteristics of low rectal cancer.

\begin{tabular}{|c|c|c|c|c|c|c|c|c|}
\hline \multirow[b]{2}{*}{ Characteristic (n) } & \multicolumn{3}{|c|}{ p53 protein expression } & \multicolumn{5}{|c|}{ p53 protein expression level } \\
\hline & Positive, $\mathrm{n}$ & Negative, $\mathrm{n}$ & P-value &,$+++ \mathrm{n}$ &,$++ \mathrm{n}$ &,$+ \mathrm{n}$ &,$- \mathrm{n}$ & P-value \\
\hline Lymph node metastasis $(\mathrm{n}=346)$ & & & 0.851 & & & & & 0.182 \\
\hline Positive & 100 & 39 & & 66 & 30 & 4 & 39 & \\
\hline Negative & 147 & 60 & & 97 & 33 & 16 & 60 & \\
\hline Histological type (n=347) & & & 0.990 & & & & & 0.056 \\
\hline Well-differentiated & 158 & 63 & & 114 & 32 & 11 & 63 & \\
\hline Poorly differentiated & 90 & 36 & & 50 & 31 & 9 & 36 & \\
\hline TNM stage $(\mathrm{n}=347)$ & & & 0.879 & & & & & 0.304 \\
\hline III-IV & 108 & 44 & & 72 & 31 & 5 & 44 & \\
\hline I-II & 140 & 55 & & 92 & 32 & 15 & 55 & \\
\hline Depth of infiltration $(n=347)$ & & & 0.254 & & & & & 0.165 \\
\hline $\mathrm{T} 3+\mathrm{T} 4$ & 127 & 57 & & 82 & 37 & 7 & 57 & \\
\hline $\mathrm{T} 1+\mathrm{T} 2$ & 122 & 47 & & 83 & 26 & 13 & 42 & \\
\hline Growth mode ( $\mathrm{n}=347)$ & & & 0.384 & & & & & 0.308 \\
\hline Nested growth & 143 & 52 & & 93 & 41 & 9 & 52 & \\
\hline Infiltration growth & 105 & 47 & & 71 & 22 & 11 & 47 & \\
\hline Vascular cancer embolus ( $\mathrm{n}=336)$ & & & 0.368 & & & & & 0.672 \\
\hline Positive & 58 & 27 & & 38 & 17 & 3 & 27 & \\
\hline Negative & 184 & 67 & & 124 & 45 & 14 & 67 & \\
\hline Extranodal implantation $(\mathrm{n}=305)$ & & & 0.507 & & & & & 0.685 \\
\hline Positive & 13 & 7 & & 10 & 3 & 0 & 7 & \\
\hline Negative & 205 & 80 & & 137 & 52 & 15 & 80 & \\
\hline Ganglion violation $(\mathrm{n}=307)$ & & & 0.595 & & & & & 0.260 \\
\hline Positive & 163 & 67 & & 111 & 43 & 8 & 67 & \\
\hline Negative & 57 & 20 & & 37 & 13 & 7 & 20 & \\
\hline $\begin{array}{l}\text { Peripheral lymphatic infiltration } \\
(\mathrm{n}=326)\end{array}$ & & & 0.454 & & & & & 0.829 \\
\hline Positive & 214 & 87 & & 146 & 52 & 15 & 87 & \\
\hline Negative & 16 & 9 & & 10 & 5 & 1 & 9 & \\
\hline
\end{tabular}

TNM, Tumor-Node-Metastasis.

with paraffin and cut into $4-\mu \mathrm{m}$ sections. Immunohistochemical staining was performed using Ultra Sensitive ${ }^{\mathrm{TM}}$ SP kit (cat. no. KIT-9709/9719; Maixin, Fuzhou, China) according to the manufacturer's protocol. Sections were deparaffinized and rehydrated through ethanol gradient $(100,95$ and $75 \%$ ethanol for 5 min each), incubated in $10 \mathrm{mM}$ citrate buffer ( $\mathrm{pH} \mathrm{6.0)}$ and heated in a microwave oven for $5 \mathrm{~min}$. After cooling, slides were incubated with blocker of endogenous peroxidase activity (buffer $\mathrm{A}$ in the kit) at room temperature for $1 \mathrm{~h}$, and blocked with normal goat serum (one drop; buffer B in the kit) for $30 \mathrm{~min}$ at room temperature. Sections were washed with PBS, incubated with anti-p53 rabbit polyclonal antibody (1:100; cat. no. ab131442; Abcam, Cambridge, UK) for $1 \mathrm{~h} \mathrm{r}$ at room temperature, with biotin-conjugated secondary antibody (one drop, buffer $\mathrm{C}$ in the kit) for $10 \mathrm{~min}$ at room temperature, and with HPR-Streptomycin (one drop, buffer D in the kit) for $10 \mathrm{~min}$ at room temperature. Signal was visualized with the 3'-diaminobenzidine visualization kit. (cat. no. dab-0031;
Fuzhou Maixin Biotech Co., Ltd.). Slides were observed with an inverted microscopy (Olympus Corporation, Tokyo, Japan).

p53 protein expression was independently read and scored by two pathologists, in accordance with the double-blind principle. A senior pathologist was consulted with regard to inconsistent scores in order to arrive at a consensus. Positive p53 protein expression was located in the nuclei of cancer cells and appeared as stronger brown granules under a microscope with high magnification (x40). Subsequently, the positive p53 protein expression area was detected under a microscope with low magnification (x10). A total of 10 fields of each slide were randomly selected under a microscope with high magnification and 100 cancer cells were counted in each field. The percentage of cancer cells with positive p53 protein expression was calculated. The scores for positive p53 expression were determined according to the percentage of p53-positive cells in each sample as follows: Negative, $<10 \%$; positive,$+ 10-30 \%$ ); ,$++ 30-50 \%$; and,$+++ 50-100 \%$. 
Table VI. Stratified association analysis between p53 protein expression and characteristics of low rectal cancer.

A, Male sex

\begin{tabular}{|c|c|c|c|c|c|c|c|c|}
\hline \multirow[b]{2}{*}{ Characteristic } & \multicolumn{3}{|c|}{ p53 protein expression } & \multicolumn{5}{|c|}{ p53 protein expression level } \\
\hline & Positive, $\mathrm{n}$ & Negative, $\mathrm{n}$ & P-value &,$+++ \mathrm{n}$ &,$++ \mathrm{n}$ &,$+ \mathrm{n}$ &,$- \mathrm{n}$ & $\mathrm{P}$-value \\
\hline Lymph node metastasis & & & 0.412 & & & & & 0.567 \\
\hline Positive & 57 & 26 & & 42 & 14 & 1 & 26 & \\
\hline Negative & 88 & 31 & & 60 & 22 & 5 & 31 & \\
\hline Histological type & & & 0.171 & & & & & 0.136 \\
\hline Well-differentiated & 97 & 32 & & 73 & 19 & 4 & 32 & \\
\hline Poorly differentiated & 49 & 25 & & 30 & 17 & 2 & 25 & \\
\hline \multicolumn{9}{|l|}{ TNM stage } \\
\hline III-IV & 62 & 28 & & 48 & 13 & 1 & 28 & \\
\hline I-II & 84 & 29 & & 55 & 23 & 5 & 29 & \\
\hline Depth of infiltration & & & 0.755 & & & & & 0.317 \\
\hline $\mathrm{T} 3+\mathrm{T} 4$ & 81 & 33 & & 54 & 24 & 2 & 33 & \\
\hline $\mathrm{T} 1+\mathrm{T} 2$ & 65 & 24 & & 49 & 12 & 4 & 24 & \\
\hline Growth mode & & & 0.857 & & & & & 0.057 \\
\hline Nested growth & 84 & 32 & & 57 & 26 & 1 & 32 & \\
\hline Infiltration growth & 62 & 25 & & 46 & 10 & 5 & 25 & \\
\hline Vascular cancer embolus & & & 0.506 & & & & & 0.447 \\
\hline Positive & 35 & 16 & & 22 & 12 & 1 & 16 & \\
\hline Negative & 108 & 39 & & 80 & 23 & 4 & 39 & \\
\hline Extranodal implantation & & & 0.786 & & & & & 0.934 \\
\hline Positive & 9 & 4 & & 7 & 2 & 0 & 4 & \\
\hline Negative & 120 & 45 & & 85 & 30 & 4 & 45 & \\
\hline Ganglion violation & & & 0.335 & & & & & 0.335 \\
\hline Positive & 98 & 40 & & 68 & 27 & 2 & 40 & \\
\hline Negative & 33 & 9 & & 25 & 6 & 2 & 9 & \\
\hline $\begin{array}{l}\text { Peripheral lymphatic } \\
\text { infiltration }\end{array}$ & & & 0.325 & & & & & 0.582 \\
\hline Positive & 126 & 49 & & 91 & 6 & 2 & 49 & \\
\hline Negative & 9 & 6 & & 8 & 30 & 4 & 6 & \\
\hline
\end{tabular}

B, Female sex

\begin{tabular}{|c|c|c|c|c|c|c|c|c|}
\hline \multirow[b]{2}{*}{ Characteristic } & \multicolumn{3}{|c|}{ p53 protein expression } & \multicolumn{5}{|c|}{ p53 protein expression level } \\
\hline & Positive, $\mathrm{n}$ & Negative, $\mathrm{n}$ & P-value &,$+++ \mathrm{n}$ &,$++ \mathrm{n}$ &,$+ \mathrm{n}$ &,$- \mathrm{n}$ & P-value \\
\hline Lymph node metastasis & & & 0.210 & & & & & 0.054 \\
\hline Positive & 43 & 13 & & 24 & 16 & 3 & 13 & \\
\hline Negative & 59 & 29 & & 37 & 11 & 11 & 29 & \\
\hline Histological type & & & 0.112 & & & & & 0.104 \\
\hline Well-differentiated & 61 & 31 & & 41 & 13 & 7 & 31 & \\
\hline Poorly differentiated & 41 & 11 & & 20 & 14 & 7 & 11 & \\
\hline TNM stage & & & & & & & & \\
\hline III-IV & 46 & 16 & & 24 & 18 & 4 & 16 & \\
\hline I-II & 56 & 26 & & 37 & 9 & 10 & 26 & \\
\hline Depth of infiltration & & & 0.155 & & & & & 0.458 \\
\hline $\mathrm{T} 3+\mathrm{T} 4$ & 45 & 24 & & 27 & 13 & 5 & 24 & \\
\hline $\mathrm{T} 1+\mathrm{T} 2$ & 57 & 18 & & 34 & 14 & 9 & 18 & \\
\hline Growth mode & & & 0.262 & & & & & 0.717 \\
\hline Nested growth & 59 & 20 & & 36 & 15 & 8 & 20 & \\
\hline Infiltration growth & 43 & 22 & & 25 & 12 & 6 & 22 & \\
\hline
\end{tabular}


Table VI. Continued.

\begin{tabular}{|c|c|c|c|c|c|c|c|c|}
\hline \multirow[b]{2}{*}{ Characteristic } & \multicolumn{3}{|c|}{ p53 protein expression } & \multicolumn{5}{|c|}{ p53 protein expression level } \\
\hline & Positive, $\mathrm{n}$ & Negative, $\mathrm{n}$ & P-value &,$+++ \mathrm{n}$ &,$++ \mathrm{n}$ &,$+ \mathrm{n}$ &,$- \mathrm{n}$ & P-value \\
\hline Vascular cancer embolus & & & 0.542 & & & & & 0.716 \\
\hline Positive & 23 & 11 & & 16 & 5 & 2 & 11 & \\
\hline Negative & 76 & 28 & & 44 & 22 & 10 & 28 & \\
\hline Extranodal implantation & & & 0.442 & & & & & 0.773 \\
\hline Positive & 4 & 3 & & 3 & 1 & 0 & 3 & \\
\hline Negative & 85 & 35 & & 52 & 22 & 11 & 35 & \\
\hline Ganglion violation & & & 0.819 & & & & & 0.423 \\
\hline Positive & 65 & 27 & & 43 & 16 & 6 & 27 & \\
\hline Negative & 24 & 11 & & 12 & 7 & 5 & 11 & \\
\hline $\begin{array}{l}\text { Peripheral lymphatic } \\
\text { infiltration }\end{array}$ & & & 0.992 & & & & & 0.294 \\
\hline Positive & 88 & 38 & & 55 & 22 & 11 & 38 & \\
\hline Negative & 7 & 3 & & 2 & 4 & 1 & 3 & \\
\hline
\end{tabular}

C, Age $\geq 60$ years

\begin{tabular}{|c|c|c|c|c|c|c|c|c|}
\hline \multirow[b]{2}{*}{ Characteristic } & \multicolumn{3}{|c|}{ p53 protein expression } & \multicolumn{5}{|c|}{ p53 protein expression level } \\
\hline & Positive, $\mathrm{n}$ & Negative, $\mathrm{n}$ & P-value &,$+++ \mathrm{n}$ &,$++ \mathrm{n}$ &,$+ \mathrm{n}$ &,$- n$ & P-value \\
\hline Lymph node metastasis & & & 0.212 & & & & & 0.166 \\
\hline Positive & 50 & 23 & & 33 & 16 & 1 & 23 & \\
\hline Negative & 95 & 29 & & 64 & 22 & 9 & 29 & \\
\hline Histological type & & & 0.127 & & & & & 0.002 \\
\hline Well-differentiated & 96 & 28 & & 73 & 16 & 7 & 28 & \\
\hline Poorly differentiated & 50 & 24 & & 25 & 22 & 3 & 24 & \\
\hline TNM stage & & & 0.073 & & & & & 0.049 \\
\hline III-IV & 55 & 27 & & 36 & 18 & 1 & 27 & \\
\hline I-II staging & 91 & 25 & & 62 & 20 & 9 & 25 & \\
\hline Depth of infiltration & & & 0.130 & & & & & 0.034 \\
\hline $\mathrm{T} 3+\mathrm{T} 4$ & 72 & 32 & & 44 & 25 & 3 & 32 & \\
\hline $\mathrm{T} 1+\mathrm{T} 2$ & 74 & 20 & & 54 & 13 & 7 & 20 & \\
\hline Growth mode & & & 0.729 & & & & & 0.227 \\
\hline Nested growth & 83 & 31 & & 55 & 25 & 3 & 31 & \\
\hline Infiltration growth & 63 & 21 & & 43 & 13 & 7 & 21 & \\
\hline Vascular cancer embolus & & & 0.158 & & & & & 0.482 \\
\hline Positive & 29 & 15 & & 20 & 8 & 1 & 15 & \\
\hline Negative & 114 & 35 & & 77 & 29 & 8 & 35 & \\
\hline Extranodal implantation & & & 0.199 & & & & & 0.529 \\
\hline Positive & 5 & 4 & & 3 & 2 & 0 & 4 & \\
\hline Negative & 125 & 42 & & 85 & 32 & 8 & 42 & \\
\hline Ganglion violation & & & 0.107 & & & & & 0.205 \\
\hline Positive & 93 & 38 & & 63 & 26 & 4 & 38 & \\
\hline Negative & 39 & 8 & & 26 & 9 & 4 & 8 & \\
\hline $\begin{array}{l}\text { Peripheral lymphatic } \\
\text { infiltration }\end{array}$ & & & 0.336 & & & & & 0.551 \\
\hline Positive & 130 & 46 & & 90 & 33 & 7 & 46 & \\
\hline Negative & 6 & 4 & & 4 & 1 & 1 & 4 & \\
\hline
\end{tabular}


Table VI. Continued.

$\mathrm{D}$, Age $<60$ years

\begin{tabular}{|c|c|c|c|c|c|c|c|c|}
\hline \multirow[b]{2}{*}{ Characteristic } & \multicolumn{3}{|c|}{ p53 protein expression } & \multicolumn{5}{|c|}{ p53 protein expression level } \\
\hline & Positive, $\mathrm{n}$ & Negative, $\mathrm{n}$ & P-value &,$+++ \mathrm{n}$ &,$++ \mathrm{n}$ &,$+ \mathrm{n}$ &,$- \mathrm{n}$ & P-value \\
\hline Lymph node metastasis & & & 0.087 & & & & & 0.166 \\
\hline Positive & 50 & 16 & & 33 & 14 & 3 & 16 & \\
\hline Negative & 52 & 31 & & 33 & 11 & 7 & 31 & \\
\hline Histological type & & & 0.103 & & & & & 0.184 \\
\hline Well-differentiated & 62 & 35 & & 41 & 16 & 4 & 35 & \\
\hline Poorly differentiated & 40 & 12 & & 25 & 9 & 6 & 12 & \\
\hline TNM stage & & & 0.073 & & & & & 0.245 \\
\hline III-IV & 53 & 17 & & 36 & 13 & 4 & 17 & \\
\hline I-II & 49 & 30 & & 30 & 12 & 6 & 30 & \\
\hline Depth of infiltration & & & 0.977 & & & & & 0.760 \\
\hline $\mathrm{T} 3+\mathrm{T} 4$ & 54 & 25 & & 37 & 12 & 4 & 25 & \\
\hline $\mathrm{T} 1+\mathrm{T} 2$ & 48 & 22 & & 29 & 13 & 6 & 22 & \\
\hline Growth mode & & & 0.107 & & & & & 0.375 \\
\hline Nested growth & 60 & 21 & & 38 & 16 & 6 & 21 & \\
\hline Infiltration growth & 42 & 26 & & 28 & 9 & 4 & 26 & \\
\hline Vascular cancer embolus & & & 0.805 & & & & & 0.855 \\
\hline Positive & 29 & 12 & & 18 & 9 & 2 & 12 & \\
\hline Negative & 20 & 32 & & 47 & 16 & 6 & 32 & \\
\hline Extranodal implantation & & & 0.737 & & & & & 0.585 \\
\hline Positive & 8 & 3 & & 7 & 1 & 0 & 3 & \\
\hline Negative & 80 & 38 & & 52 & 20 & 7 & 38 & \\
\hline Ganglion violation & & & 0.270 & & & & & 0.358 \\
\hline Positive & 70 & 29 & & 48 & 17 & 4 & 29 & \\
\hline Negative & 18 & 12 & & 11 & 4 & 3 & 12 & \\
\hline $\begin{array}{l}\text { Peripheral lymphatic } \\
\text { infiltration }\end{array}$ & & & 0.967 & & & & & 0.554 \\
\hline Positive & 84 & 41 & & 56 & 19 & 8 & 41 & \\
\hline Negative & 10 & 5 & & 6 & 4 & 0 & 5 & \\
\hline
\end{tabular}

E, Smoker

\begin{tabular}{|c|c|c|c|c|c|c|c|c|}
\hline \multirow[b]{2}{*}{ Characteristic } & \multicolumn{3}{|c|}{ p53 protein expression } & \multicolumn{5}{|c|}{ p53 protein expression level } \\
\hline & Positive, $\mathrm{n}$ & Negative, $\mathrm{n}$ & P-value &,$+++ \mathrm{n}$ &,$++ \mathrm{n}$ &,$+ \mathrm{n}$ &,$- \mathrm{n}$ & P-value \\
\hline Lymph node metastasis & & & 0.032 & & & & & 0.095 \\
\hline Positive & 26 & 16 & & 18 & 7 & 1 & 16 & \\
\hline Negative & 54 & 13 & & 40 & 8 & 5 & 13 & \\
\hline Histological type & & & 0.198 & & & & & 0.608 \\
\hline Well-differentiated & 58 & 17 & & 43 & 10 & 4 & 17 & \\
\hline Poorly differentiated & 23 & 12 & & 16 & 5 & 2 & 12 & \\
\hline TNM stage & & & & & & & & \\
\hline III-IV & 31 & 16 & & 24 & 6 & 1 & 16 & \\
\hline I-II & 50 & 13 & & 35 & 9 & 5 & 13 & \\
\hline Depth of infiltration & & & 0.135 & & & & & 0.375 \\
\hline $\mathrm{T} 3+\mathrm{T} 4$ & 40 & 19 & & 30 & 7 & 2 & 19 & \\
\hline $\mathrm{T} 1+\mathrm{T} 2$ & 41 & 10 & & 29 & 8 & 4 & 10 & \\
\hline
\end{tabular}


Table VI. Continued.

\begin{tabular}{|c|c|c|c|c|c|c|c|c|}
\hline \multirow[b]{2}{*}{ Characteristic } & \multicolumn{3}{|c|}{ p53 protein expression } & \multicolumn{5}{|c|}{ p53 protein expression level } \\
\hline & Positive, $\mathrm{n}$ & Negative, $\mathrm{n}$ & P-value &,$+++ \mathrm{n}$ &,$++ \mathrm{n}$ &,$+ \mathrm{n}$ &,$- \mathrm{n}$ & P-value \\
\hline Growth mode & & & 0.608 & & & & & 0.381 \\
\hline Nested growth & 43 & 17 & & 29 & 11 & 3 & 17 & \\
\hline Infiltration growth & 38 & 12 & & 30 & 4 & 3 & 12 & \\
\hline Vascular cancer embolus & & & 0.882 & & & & & 0.114 \\
\hline Positive & 18 & 6 & & 10 & 7 & 1 & 6 & \\
\hline Negative & 61 & 22 & & 48 & 8 & 4 & 22 & \\
\hline Extranodal implantation & & & 0.226 & & & & & 0.578 \\
\hline Positive & 4 & 0 & & 3 & 1 & 0 & 0 & \\
\hline Negative & 70 & 26 & & 51 & 13 & 5 & 26 & \\
\hline Ganglion violation & & & 0.625 & & & & & 0.392 \\
\hline Positive & 54 & 20 & & 40 & 11 & 2 & 20 & \\
\hline Negative & 21 & 6 & & 14 & 4 & 3 & 6 & \\
\hline $\begin{array}{l}\text { Peripheral lymphatic } \\
\text { infiltration }\end{array}$ & & & 0.269 & & & & & 0.642 \\
\hline Positive & 77 & 26 & & 56 & 15 & 5 & 26 & \\
\hline Negative & 2 & 2 & & 2 & 0 & 0 & 2 & \\
\hline
\end{tabular}

F, Non-smoker

\begin{tabular}{|c|c|c|c|c|c|c|c|c|}
\hline \multirow[b]{2}{*}{ Characteristic } & \multicolumn{3}{|c|}{ p53 protein expression } & \multicolumn{5}{|c|}{ p53 protein expression level } \\
\hline & Positive, $\mathrm{n}$ & Negative, $\mathrm{n}$ & P-value &,$+++ \mathrm{n}$ &,$++ \mathrm{n}$ &,$+ \mathrm{n}$ &,$- \mathrm{n}$ & P-value \\
\hline Lymph node metastasis & & & 0.102 & & & & & 0.109 \\
\hline Positive & 74 & 23 & & 48 & 23 & 3 & 23 & \\
\hline Negative & 93 & 47 & & 57 & 25 & 11 & 47 & \\
\hline Histological type & & & 0.400 & & & & & 0.047 \\
\hline Well-differentiated & 100 & 46 & & 71 & 22 & 7 & 46 & \\
\hline Poorly-differentiated & 67 & 24 & & 34 & 26 & 7 & 24 & \\
\hline TNM stage & & & & & & & & \\
\hline III-IV & 77 & 28 & & 48 & 25 & 4 & 28 & \\
\hline I-II & 90 & 42 & & 57 & 23 & 10 & 42 & \\
\hline Depth of infiltration & & & 0.695 & & & & & 0.237 \\
\hline $\mathrm{T} 3+\mathrm{T} 4$ & 86 & 38 & & 51 & 30 & 5 & 38 & \\
\hline $\mathrm{T} 1+\mathrm{T} 2$ & 81 & 32 & & 54 & 18 & 9 & 32 & \\
\hline Growth mode & & & 0.161 & & & & & 0.284 \\
\hline Nested growth & 100 & 35 & & 64 & 30 & 6 & 35 & \\
\hline Infiltration growth & 67 & 35 & & 41 & 18 & 8 & 35 & \\
\hline Vascular cancer embolus & & & 0.259 & & & & & 0.529 \\
\hline Positive & 40 & 21 & & 28 & 10 & 2 & 21 & \\
\hline Negative & 123 & 45 & & 76 & 37 & 10 & 45 & \\
\hline Extranodal implantation & & & 0.202 & & & & & 0.478 \\
\hline Positive & 9 & 7 & & 7 & 2 & 0 & 7 & \\
\hline Negative & 135 & 54 & & 86 & 39 & 10 & 54 & \\
\hline Ganglion violation & & & 0.774 & & & & & 0.676 \\
\hline Positive & 109 & 47 & & 71 & 32 & 6 & 47 & \\
\hline Negative & 36 & 14 & & 23 & 9 & 4 & 14 & \\
\hline $\begin{array}{l}\text { Peripheral lymphatic } \\
\text { infiltration }\end{array}$ & & & 0.812 & & & & & 0.912 \\
\hline Positive & 137 & 61 & & 90 & 37 & 10 & 61 & \\
\hline Negative & 14 & 7 & & 8 & 5 & 1 & 7 & \\
\hline
\end{tabular}


Table VI. Continued.

G, Alcohol consumption

\begin{tabular}{|c|c|c|c|c|c|c|c|c|}
\hline \multirow[b]{2}{*}{ Characteristic } & \multicolumn{3}{|c|}{ p53 protein expression } & \multicolumn{5}{|c|}{ p53 protein expression level } \\
\hline & Positive, $\mathrm{n}$ & Negative, $\mathrm{n}$ & P-value &,$+++ \mathrm{n}$ &,$++ \mathrm{n}$ &,$+ \mathrm{n}$ &,$- \mathrm{n}$ & P-value \\
\hline Lymph node metastasis & & & 0.816 & & & & & 0.499 \\
\hline Positive & 11 & 4 & & 7 & 4 & 0 & 4 & \\
\hline Negative & 21 & 9 & & 16 & 3 & 1 & 9 & \\
\hline Histological type & & & 0.458 & & & & & 0.243 \\
\hline Well-differentiated & 24 & 8 & & 19 & 4 & 0 & 8 & \\
\hline Poorly differentiated & 9 & 5 & & 5 & 3 & 1 & 5 & \\
\hline TNM stage & & & & & & & & \\
\hline III-IV & 12 & 4 & & 8 & 4 & 0 & 4 & \\
\hline I-II & 21 & 9 & & 16 & 3 & 1 & 9 & \\
\hline Depth of infiltration & & & 0.818 & & & & & 0.718 \\
\hline $\mathrm{T} 3+\mathrm{T} 4$ & 19 & 7 & & 14 & 4 & 0 & 7 & \\
\hline $\mathrm{T} 1+\mathrm{T} 2$ & 14 & 6 & & 10 & 3 & 1 & 6 & \\
\hline Growth mode & & & 0.818 & & & & & 0.575 \\
\hline Nested growth & 19 & 7 & & 14 & 5 & 0 & 7 & \\
\hline Infiltration growth & 14 & 6 & & 10 & 2 & 1 & 6 & \\
\hline Vascular cancer embolus & & & 0.452 & & & & & 0.363 \\
\hline Positive & 8 & 2 & & 5 & 3 & 0 & 2 & \\
\hline Negative & 23 & 11 & & 18 & 3 & 1 & 11 & \\
\hline Extranodal implantation & & & 0.220 & & & & & 0.496 \\
\hline Positive & 3 & 0 & & 2 & 1 & 0 & 0 & \\
\hline Negative & 25 & 13 & & 19 & 4 & 1 & 13 & \\
\hline Ganglion violation & & & 0.226 & & & & & 0.130 \\
\hline Positive & 23 & 8 & & 17 & 5 & 0 & 8 & \\
\hline Negative & 6 & 5 & & 5 & 0 & 1 & 5 & \\
\hline $\begin{array}{l}\text { Peripheral lymphatic } \\
\text { infiltration }\end{array}$ & & & 0.314 & & & & & 0.613 \\
\hline Positive & 25 & 13 & & 19 & 4 & 1 & 13 & \\
\hline Negative & 2 & 0 & & 2 & 0 & 0 & 0 & \\
\hline
\end{tabular}

H, No alcohol consumption

\begin{tabular}{|c|c|c|c|c|c|c|c|c|}
\hline \multirow[b]{2}{*}{ Characteristic } & \multicolumn{3}{|c|}{ p53 protein expression } & \multicolumn{5}{|c|}{ p53 protein expression level } \\
\hline & Positive, $\mathrm{n}$ & Negative, $\mathrm{n}$ & P-value &,$+++ \mathrm{n}$ &,$++ \mathrm{n}$ &,$+ \mathrm{n}$ &,$- \mathrm{n}$ & P-value \\
\hline Lymph node metastasis & & & 0.912 & & & & & 0.275 \\
\hline Positive & 89 & 35 & & 59 & 26 & 4 & 35 & \\
\hline Negative & 126 & 51 & & 81 & 30 & 15 & 51 & \\
\hline Histological type & & & 0.792 & & & & & 0.127 \\
\hline Well-differentiated & 134 & 55 & & 95 & 28 & 11 & 55 & \\
\hline Poorly differentiated & 81 & 31 & & 45 & 28 & 8 & 31 & \\
\hline \multicolumn{9}{|l|}{ TNM stage } \\
\hline III-IV & 96 & 40 & & 64 & 27 & 5 & 40 & \\
\hline I-II & 119 & 46 & & 76 & 29 & 14 & 46 & \\
\hline Depth of infiltration & & & 0.189 & & & & & 0.166 \\
\hline $\mathrm{T} 3+\mathrm{T} 4$ & 107 & 50 & & 67 & 33 & 7 & 50 & \\
\hline $\mathrm{T} 1+\mathrm{T} 2$ & 108 & 36 & & 73 & 23 & 12 & 36 & \\
\hline Growth mode & & & 0.398 & & & & & 0.455 \\
\hline Nested growth & 124 & 45 & & 79 & 36 & 9 & 45 & \\
\hline Infiltration growth & 91 & 41 & & 61 & 20 & 10 & 41 & \\
\hline
\end{tabular}


Table VI. Continued.

\begin{tabular}{|c|c|c|c|c|c|c|c|c|}
\hline \multirow[b]{2}{*}{ Characteristic } & \multicolumn{3}{|c|}{ p53 protein expression } & \multicolumn{5}{|c|}{ p53 protein expression level } \\
\hline & Positive, $\mathrm{n}$ & Negative, $\mathrm{n}$ & P-value &,$+++ \mathrm{n}$ &,$++ \mathrm{n}$ &,$+ \mathrm{n}$ &,$- \mathrm{n}$ & P-value \\
\hline Vascular cancer embolus & & & 0.209 & & & & & 0.608 \\
\hline Positive & 50 & 25 & & 33 & 14 & 3 & 25 & \\
\hline Negative & 161 & 56 & & 106 & 42 & 13 & 56 & \\
\hline Extranodal implantation & & & 0.212 & & & & & 0.461 \\
\hline Positive & 10 & 7 & & 8 & 2 & 0 & 7 & \\
\hline Negative & 180 & 67 & & 118 & 48 & 14 & 67 & \\
\hline Ganglion violation & & & 0.277 & & & & & 0.349 \\
\hline Positive & 140 & 59 & & 94 & 38 & 8 & 59 & \\
\hline Negative & 51 & 15 & & 32 & 13 & 6 & 15 & \\
\hline $\begin{array}{l}\text { Peripheral lymphatic } \\
\text { infiltration }\end{array}$ & & & 0.265 & & & & & 0.599 \\
\hline Positive & 189 & 74 & & 127 & 48 & 14 & 74 & \\
\hline Negative & 14 & 9 & & 8 & 5 & 1 & 9 & \\
\hline
\end{tabular}

Statistical analysis. Statistical analysis was performed using SPSS 20.0 software (IBM Corp., Armonk, NY, USA). Independent sample t-test was used to compare the differences between two groups, and one-way ANOVA followed by Tukey post-hoc analysis was used to compare the differences among multiple groups $(>2)$. Parameters that reflected the behavior and prognosis of LRC in each genotype were represented by hazard ratios (HR) and 95\% confidence intervals (CIs). The HR values were calculated by multivariate Cox proportional hazards regression analysis. The $\chi^{2}$ test was used to evaluate the association between TP53 gene polymorphism and p53 protein expression, or between $\mathrm{p} 53$ protein expression and the clinical pathological parameters of LRC. The log-rank test was used to compare the survival times between the groups. $\mathrm{P}<0.05$ was considered to indicate a statistically significant difference.

\section{Results}

Association between TP53 gene polymorphism and p53 protein expression. To study the influence of TP53 gene polymorphism on the protein expression of p53, five polymorphic loci of the TP53 gene (rs1042522, rs12947788, rs1625895, rs2909430 and rs12951053) and the protein expression level of p53 in the 347 patients diagnosed with LRC were detected. Results revealed that the TP53 rs1042522 polymorphism was associated with $\mathrm{p} 53$ protein expression [CG (heterozygous) vs. GG (mutant), $\mathrm{P}=0.027$; $\mathrm{CC}$ (wide-type) + CG vs. GG, $\mathrm{P}=0.032$ ], indicating that positive $\mathrm{p} 53$ protein expression was significantly higher compared with other genotypes in the heterozygous and dominant models (Table III and Fig. 1). The other four polymorphic loci were not identified to be associated with $\mathrm{p} 53$ protein expression.

Protein expression of p53 is associated with the biological characteristics of $L R C$. In the present study, the association between time survival and clinicopathological parameters of LRC, including TNM classification, depth of invasion, histological type, paracancerous lymphocyte infiltration, ganglion infiltration, vascular cancer embolus, lymph node metastasis and extranodal implantation status were analyzed (Table IV). The $\chi^{2}$ test revealed that the protein expression of p53 was not significantly associated with the clinicopathological parameters in the whole population (Table V). However, following stratification of the patients by classic risk factors, including sex, age, smoking status and alcohol consumption, the results revealed a significant association between the protein expression of p53 and the clinicopathological parameters of LRC. In female patients, the protein expression of p53 in stage III-IV was significantly higher compared with that in stage I-II $(\mathrm{P}=0.044)$. Furthermore, in patients with an age $\geq 60$ years, histological type, TNM stage and depth of tumor invasion were all associated with the protein expression of $\mathrm{p} 53$ ( $\mathrm{P}=0.002, \mathrm{P}=0.049$ and $\mathrm{P}=0.034$, respectively). The protein expression of p53 was significantly higher in poorly-differentiated tumors compared with well-differentiated tumors, in stage III-IV compared with stage I-II, and in the T3-4 stage compared with the T1-2 stage. In patients with a history of smoking, the p53 protein expression was significantly associated with the occurrence of lymph node metastasis $(\mathrm{P}=0.032)$. In contrast to smokers, the $\mathrm{p} 53$ protein expression level in poorly-differentiated tumors was significantly higher compared with well-differentiated tumors in non-smokers $(\mathrm{P}=0.047$; Table VI).

p53 protein expression is associated with the prognosis of $L R C$. To further determine whether $\mathrm{p} 53$ protein expression is an independent prognostic factor for LRC, univariate and multivariate Cox proportional hazards regression analyses were conducted (Table VII). Univariate survival analysis revealed a significant association between the protein 

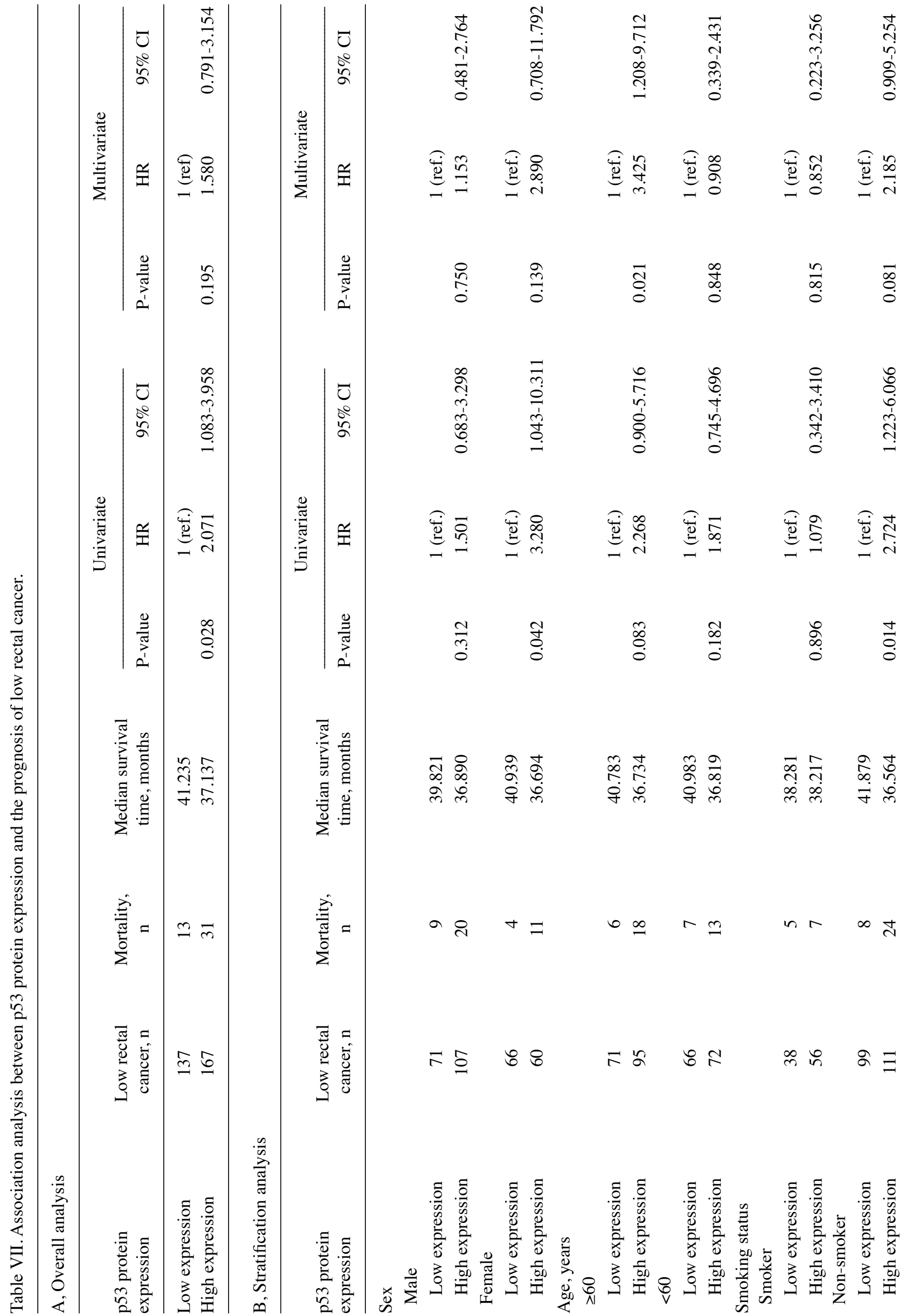
expression of p53 and the OS for LRC; the survival time of patients with low p53 expression was significantly longer compared with that of patients with high p53 expression [hazard ratio (HR), 2.071; 95\% CI, 1.083-3.958; P=0.028]. However, the multivariate survival analysis revealed that the protein expression level of p53 was no longer associated with the survival time in all patients with LRC (HR, 1.580; 95\% CI, 0.791-3.154; $\mathrm{P}=0.195$ ) (Table VII). Following stratification of the patients according to risk factors of LRC, including sex, age, smoking status and drinking status, the results revealed that the survival time of patients with low p53 protein expression was significantly longer compared with patients with high p53 protein expression in female patients (HR, 3.280; 95\% CI, $1.043-10.311 ; \mathrm{P}=0.042)$ and non-smokers (HR, 2.724; 95\% CI, 1.223-6.066; $\mathrm{P}=0.014)$. Multivariate analysis for patients with an age $\geq 60$ years revealed that patients with low $\mathrm{p} 53$ protein expression had a longer survival time compared with patients with high $\mathrm{p} 53$ protein expression $(\mathrm{P}=0.021$; $\mathrm{HR}, 3.425 ; 95 \%$ CI, 1.208-9.712).

\section{Discussion}

As a subtype of colorectal cancer at a special anatomical site, LRC is characterized by its specific biological behavior. TP53 is one of the most important cancer suppressor genes, and structural variation and abnormal expression of p53 have been identified to be associated with numerous cancer types (22-25). However, the associations between TP53 gene polymorphisms and protein expression, and the association of p53 protein expression with the biological behavior and prognosis of LRC have not been clearly investigated. Understanding these associations is important for the preoperative assessment of LRC and the development of individualized treatments. The present study investigated the associations between TP53 gene polymorphisms and p53 protein expression, and the associations between $\mathrm{p} 53$ protein expression and the biological behavior and prognosis of LRC. The overall aim was to address the role of TP53 gene polymorphisms and p53 protein expression in the biological behavior and prognosis of LRC.

Genetic polymorphisms are a common genetic variation, which may affect the expression of proteins and protein function (26-29). In the present study, the associations between the five most common TP53 SNPs (rs1042522, rs12947788, rs1625895, rs2909430 and rs12951053) and p53 protein expression were evaluated. The results revealed that the TP53 rs1042522 polymorphism was associated with p53 protein expression, which was evidenced by the significantly higher p53 protein expression in TP53 rs1042522 mutant carriers compared with that in the other genotypes. No associations were identified between 553 protein expression and the other TP53 SNPs. Among the five polymorphic loci selected in the present study, only rs1042522 was located in the exon region, whereas the other four polymorphic loci were located in the intron region. This indicated that the rs 1042522 polymorphism may be present in the coding sequence of the TP53 gene, affecting therefore the protein expression of p53 (30). However, this does not indicate that other polymorphisms are not functionally important, since SNPs that are not located in protein coding regions 
may affect other processes, including gene splicing, which requires further investigation.

Although previous studies have been conducted to investigate the protein expression of p53 and its association with the clinical biological behavior and prognosis of colorectal cancer, results from these studies have been inconsistent. Furthermore, to the best of our knowledge, systematic studies focusing on LRC have not previously been performed. Therefore, the association between p53 expression and LRC at $6-8 \mathrm{~cm}$ from the anal margin was investigated in the present study. Overall analysis results revealed that there was no significant association between p53 protein expression and the clinicopathological parameters of LRC. However, following stratification analysis, an association was identified between lymphatic metastasis in smokers and p53 protein expression. Furthermore, histological type, TNM stage and tumor infiltration depth were associated with p53 expression level in patients $\geq 60$ years old. In addition, p53 expression was markedly higher in poorly-differentiated, III-IV phase or T3-4 phase tumors, and a significant association was revealed between p53 expression level and TNM stage in female patients, which was evidently higher in III-IV phase female patients. Additionally, an association was identified between p53 expression and the histological type of LRC among non-smokers.

The survival time of patients with low p53 protein expression was significantly longer in females, non-smokers and patients $\geq 60$ years old. These results indicate that $\mathrm{p} 53$ protein expression may be used as an indicator for the prognosis of LRC, particularly for patients $\geq 60$ years old, non-smokers, patients with III-IV phase tumor or female patients with T3-4 phase tumors. Although the exact mechanism requires further exploration, the current findings indicate that p53 protein expression should be regularly screened in the aforementioned subgroups of patients to enable individualized treatments that improve clinical outcomes in future clinical practice.

In conclusion, the TP53 rs1042522 polymorphism affects the $\mathrm{p} 53$ protein expression in LRC, and p53 protein expression is associated with the biological behavior and prognosis of LRC. Therefore, the TP53 rs1042522 polymorphism and p53 protein expression may serve as indicators to predict the biological behavior and prognosis of LRC.

\section{Acknowledgements}

Not applicable.

\section{Funding}

The present study was supported by the National Science and Technology Support Program (grant no. 2015BAI13B07) and the National Key R\&D Program (grant no. 2016YFC1303202).

\section{Availability of data and materials}

All data generated or analyzed during the present study are included in this published article.

\section{Authors' contributions}

YY designed the study. CX, GZ, ZW and LS collected the samples and performed the experiments. QX, ZL and JL performed the statistical analysis. GZ and QX drafted the manuscript. YY revised the manuscript. All authors approved final version of the manuscript.

\section{Ethics approval and consent to participate}

This study was approved by the Medical Ethics Committee of the First Hospital of China Medical University (Shenyang, China) and written informed consent was obtained from all participants.

\section{Patient consent for publication}

Not applicable.

\section{Competing interests}

The authors declare that they have no competing interests.

\section{References}

1. Pachler J and Wille-Jørgensen P: Quality of life after rectal resection for cancer, with or without permanent colostomy. Cochrane Database Syst Rev 12: CD004323, 2012

2. Sarver AL, Li L and Subramanian S: MicroRNA miR-183 functions as an oncogene by targeting the transcription factor EGR1 and promoting tumor cell migration. Cancer Res 70: 9570-9580, 2010.

3. Liu Y, Zhang H, Zhou K, Chen L, Xu Z, Zhong Y, Liu H, Li R, Shugart YY, Wei Q, et al: Tagging SNPs in non-homologous end-joining pathway genes and risk of glioma. Carcinogenesis 28: 1906-1913, 2007

4. Liu Y, Zhou K, Zhang H, Shugart YY, Chen L, Xu Z, Zhong Y, Liu H, Jin L, Wei Q, et al: Polymorphisms of LIG4 and XRCC4 involved in the NHEJ pathway interact to modify risk of glioma. Hum Mutat 29: 381-389, 2008.

5. Liu Y, Shete S, Etzel CJ, Scheurer M, Alexiou G, Armstrong G, Tsavachidis S, Liang FW, Gilbert M, Aldape K, et al: Polymorphisms of LIG4, BTBD2, HMGA2, and RTEL1 genes involved in the double-strand break repair pathway predict glioblastoma survival. J Clin Oncol 28: 2467-2474, 2010.

6. Soussi T and Béroud C: Assessing TP53 status in human tumours to evaluate clinical outcome. Nat Rev Cancer 1: 233-240, 2001.

7. Bénard J, Douc-Rasy S and Ahomadegbe JC: TP53 family members and human cancers. Hum Mutat 21: 182-191, 2003.

8. Li D, Suzuki H, Liu B, Morris J, Liu J, Okazaki T, Li Y, Chang P and Abbruzzese JL: DNA repair gene polymorphisms and risk of pancreatic cancer. Clin Cancer Res 15: 740-746, 2009.

9. Liu $\mathrm{H}$ and Zhou M: Evaluation of p53 gene expression and prognosis characteristics in uveal melanoma cases. Onco Targets Ther 10: 3429-3434, 2017.

10. Chava S, Mohan V, Shetty PJ, Manolla ML, Vaidya S, Khan IA, Waseem GL, Boddala P, Ahuja YR and Hasan Q: Immunohistochemical evaluation of p53, FHIT, and IGF2 gene expression in esophageal cancer. Dis Esophagus 25: 81-87, 2012.

11. Vogelstein B, Lane D and Levine AJ: Surfing the p53 network. Nature 408: 307-310, 2000.

12. Tefre T, Ryberg D, Haugen A, Nebert DW, Skaug V, Brøgger A and Børresen AL: Human CYP1A1 (cytochrome P (1)450) gene: Lack of association between the Msp I restriction fragment length polymorphism and incidence of lung cancer in a Norwegian population. Pharmacogenetics 1: 20-25, 1991.

13. Slattery ML, Samowtiz W, Ma K, Murtaugh M, Sweeney C, Levin TR and Neuhausen S: CYP1A1, cigarette smoking, and colon and rectal cancer. Am J Epidemiol 160: 842-852, 2004

14. Kiyohara C, Washio M, Horiuchi T, Asami T, Ide S, Atsumi T, Kobashi G, Takahashi H and Tada Y; Kyushu Sapporo SLE (KYSS) Study Group: Risk modification by CYP1A1 and GSTM1 polymorphisms in the association of cigarette smoking and systemic lupus erythematosus in a Japanese population. Scand J Rheumatol 41: 103-109, 2012.

15. Goodman JE, Mechanic LE, Luke BT, Ambs S, Chanock S and Harris CC: Exploring SNP-SNP interactions and colon cancer risk using polymorphism interaction analysis. Int J Cancer 118: 1790-1797, 2006. 
16. Tan XL, Nieters A, Hoffmeister M, Beckmann L, Brenner H and Chang-Claude J: Genetic polymorphisms in Tp53, nonsteroidal anti-inflammatory drugs and the risk of colorectal cancer: Evidence for gene-environment interaction? Pharmacogenet Genomics 17: 639-645, 2007.

17. Li XL, Zhou J, Chen ZR and Chng WJ: P53 mutations in colorectal cancer-molecular pathogenesis and pharmacological reactivation. World J Gastroenterol 21: 84-93, 2015.

18. Kahlenberg MS, Stoler DL, Rodriguez-Bigas MA, Weber TK, Driscoll DL, Anderson GR and Petrelli NJ: p53 tumor suppressor gene mutations predict decreased survival of patients with sporadic colorectal carcinoma. Cancer 88: 1814-1819, 2000.

19. Mulder JW, Baas IO, Polak MM, Goodman SN and Offerhaus GJ: Evaluation of p53 protein expression as a marker for long-term prognosis in colorectal carcinoma. Br J Cancer 71: 1257-1262, 1995.

20. Erhan Y, Korkut MA, Kara E, Aydede H, Sakarya A and Ilkgü O: Value of p53 protein expression and its relationship with short-term prognosis in colorectal cancer. Ann Saudi Med 22: 377-380, 2002.

21. Glimelius B, Tiret E, Cervantes A and Arnold D; ESMO Guidelines Working Group: Rectal cancer: ESMO clinical practice guidelines for diagnosis, treatment and follow-up. Ann Oncol 24 (Suppl 6): vi81-vi88, 2013.

22. Albibas AA, Rose-Zerilli MJJ, Lai C, Pengelly RJ, Lockett GA, Theaker J, Ennis S, Holloway JW and Healy E: Subclonal evolution of cancer-related gene mutations in p53 immunopositive patches in human skin. J Invest Dermatol 138: 189-198, 2018.

23. Duffy MJ, Synnott NC and Crown J: Mutant p53 as a target for cancer treatment. Eur J Cancer 83: 258-265, 2017.
24. Jen J, Lin LL, Lo FY, Chen HT, Liao SY, Tang YA, Su WC, Salgia R, Hsu CL, Huang HC, et al: Oncoprotein ZNF322A transcriptionally deregulates alpha-adducin, cyclin D1 and p53 to promote tumor growth and metastasis in lung cancer. Oncogene 36: 5219, 2017.

25. Chaudhary R, Gryder B, Woods WS, Subramanian M, Jones MF, Li XL, Jenkins LM, Shabalina SA, Mo M, Dasso M, et al: Prosurvival long noncoding RNA PINCR regulates a subset of p53 targets in human colorectal cancer cells by binding to Matrin 3. Elife 6: pii: e23244, 2017.

26. Jiang Z, Hennein L, Xu Y, Bao N, Coh P and Tao L: Elevated serum monocyte chemoattractant protein-1 levels and its genetic polymorphism is associated with diabetic retinopathy in Chinese patients with type 2 diabetes. Diabet Med 33: 84-690, 2016.

27. Zlotorynski E: Cancer biology: A Neat target of p53. Nat Rev Mol Cell Biol 18: 532, 2017.

28. Boiocchi C, Osera C, Monti MC, Ferraro OE, Govoni S, Cuccia M, Montomoli C, Pascale A and Bergamaschi R: Are Hsp70 protein expression and genetic polymorphism implicated in multiple sclerosis inflammation? J Neuroimmunol 268: 84-88, 2014.

29. Yao C, Li G, Cai M, Qian Y, Wang L, Xiao L, Thaiss F and Shi B: Expression and genetic polymorphism of necroptosis related protein RIPK1 is correlated with severe hepatic ischemia-reperfusion injury and prognosis after hepatectomy in hepatocellular carcinoma patients. Cancer Biomark 20: 23-29, 2017.

30. Naccarati A, Polakova V, Pardini B, Vodickova L, Hemminki K, Kumar R and Vodicka P: Mutations and polymorphisms in TP53 gene-an overview on the role in colorectal cancer. Mutagenesis 27: 211-218, 2012. 